\title{
Evaluation of cumulative cognitive deficits from electroconvulsive therapy
}

George G. Kirov, Laura Owen, Hazel Ballard, Adele Leighton, Kara Hannigan, Danielle Llewellyn, Valentina Escott-Price and Maria Atkins

\section{Background}

Electroconvulsive therapy (ECT) is the most effective acute treatment for severe depression, but widely held concerns about memory problems may limit its use.

\section{Aims}

To find out whether repeated or maintenance courses of ECT cause cumulative cognitive deterioration.

\section{Method}

Analysis of the results of 10 years of cognitive performance data collection from patients who have received ECT. The 199 patients had a total of 498 assessments, undertaken after a mean of 15.3 ECT sessions (range 0-186). A linear mixed-effect regression model was used, testing whether an increasing number of ECT sessions leads to deterioration in performance.

\section{Results}

The total number of previous ECT sessions had no effect on cognitive performance. The major factors affecting performance were age, followed by the severity of depression at the time of testing and the number of days since the last ECT session.

\section{Conclusions}

Repeated courses of ECT do not lead to cumulative cognitive deficits. This message is reassuring for patients, carers and prescribers who are concerned about memory problems and confusion during ECT.

\section{Declaration of interest}

None.

\section{Copyright and usage}

(c) The Royal College of Psychiatrists 2016.
Electroconvulsive therapy (ECT) is the most effective acute treatment for severe depression, ${ }^{1}$ with reported remission rates above $50 \%{ }^{2,3}$ Although some reports demonstrate even higher remission rates (such as $75 \%$ in patients with psychotic depression ${ }^{4}$ ), these could be below $50 \%$ for treatment-resistant depression or in community settings. ${ }^{5,6}$ ECT is often portrayed in mainstream media as a barbaric treatment ${ }^{7}$ and its cognitive side-effects as profound and debilitating, leading to public, patient and carer concerns. ECT does cause retrograde amnesia and acute disorientation immediately following a treatment, ${ }^{8}$ however, research has suggested that this is only a short-lived side-effect. A meta-analysis by Semkovska \& McLoughlin ${ }^{9}$ analysed the cognitive tests of 2981 patients from 84 studies, performed before and after single courses of ECT, and found that a decline in cognitive performance was limited to the first 3 days following a treatment. Patients showed no cognitive deterioration when tested 2 or more weeks after their last ECT session. This does not apply to retrograde amnesia, which was not part of this analysis, and it cannot be extended to cognitive functions that were not tested. Much less is known about the side-effects of long-term ECT, including maintenance ECT. A major concern of patients and some health professionals is that it could lead to progressive cognitive deficits, especially if given for prolonged periods of time. Small studies and case reports have addressed this question and have found no evidence to support this concern (see Discussion). Over the past 10 years we performed prospective cognitive tests on 199 patients, of whom 96 had $>12$ ECT sessions during their lifetime (the usual maximum duration of a single ECT course). We wanted to find out whether there was evidence that their cognitive performance deteriorated with the increasing number of ECT sessions.

\section{Method}

We introduced cognitive testing for ECT patients at our department in 2004, first as part of a study comparing ECT and magnetic seizure therapy. ${ }^{10}$ Influenced by the general perceptions at that time, we expected that ECT would cause a deterioration in cognitive tests. We found the tests very useful when making decisions about repeated ECT courses or maintenance ECT and when addressing patients' concerns. Therefore, we introduced these tests as part of the standard assessments of patients. More recently routine cognitive testing was recommended by the ECT Accreditation Service (ECTAS) of the Royal College of Psychiatrists. The list of nine tests that we use is presented in the Appendix. ${ }^{9,11,12}$ Tests were administered by the authors of this paper, staff at the ECT department, students or psychiatrists at Cardiff University, as part of their projects over the years. All raters were trained and supervised in administering the tests by the first author. Therefore this is a non-masked study, with all the potential biases arising from such studies. However, the tests have been administered by many people, who might have had different expectations and could not know how the results could be analysed, therefore we cannot identify any systematic bias that has operated on the scoring. All patients who were referred for ECT were offered cognitive testing. Not everybody wanted to be tested and the more impaired patients were not able to perform some or all of the tests. Tests were performed before the start of a course, within 1 week after its end and at 3-month follow-up. Over the 10 years, many patients came for repeated courses and were offered repeated testing. Some patients required maintenance ECT (for example 11 patients had $>50$ ECT sessions) and we were initially concerned that they would experience a progressive cognitive deterioration. We wanted to monitor this and introduced yearly testing of patients having maintenance ECT.

Patients had between 0 and 186 previous ECT sessions at the time of their tests. A total of 199 patients were assessed on between one and seven occasions. The number of assessments on any single test varied between 332 and 493, as some tests were introduced later and not everybody completed all tests at each assessment point as a result of refusal, time constraints or technical problems. 
We excluded patients with schizophrenia, because of their known cognitive deficits. ${ }^{13}$ Patients who were not fluent in English were not included in this analysis.

The mean age of patients at the time of their last assessment was 56.3 years (s.d. $=15.8$, range $21-88$ ) and $71.9 \%$ of them were female $(n=143)$. The majority had unipolar depression $(n=166$, $83.4 \%$ ), the remaining 33 had bipolar affective disorder, but everybody included in this analysis was treated for depressive episodes. The average number of ECT sessions that patients had prior to their tests was 15.3 (s.d. $=23.2$, range 0-186). Figure 1 shows the distribution of the numbers of ECT sessions that patients had prior to their tests.

\section{Statistical analysis}

Our aim was to evaluate whether the cumulative number of ECT sessions received during the lifetime of patients prior to testing was inversely correlated with their performance on the cognitive tests. We obtained histories of previous courses and recorded the total number of ECT sessions they had received prior to each test. As other factors can influence cognitive performance, we included the following four covariates: age, number of days since last ECT session, severity of depression and the number of times the patient had undertaken the test previously. Age is an obvious confounder, as cognitive performance declines with age. It has been shown that cognitive performance is worse in the first 3 days after an ECT session and tends to improve with time. ${ }^{9}$ We recorded the number of days since the last ECT session (for those who had no previous ECT sessions, we entered an arbitrary, but very large number of 10000 days (about 30 years), in order to keep these assessments in the analysis). As these data were extremely skewed, we log-transformed the number of days since the last ECT. As our cognitive testing always includes an objective measure of the severity of depression (the 24-item Hamilton Rating Scale for Depression, HRSD-24), ${ }^{14}$ we included these scores as a covariate, expecting that patients who were more severely depressed might perform worse. The number of times a test had been undertaken previously by a patient was also included as a covariate in order to control for the possibility that patients improve their performance with practice. As some patients had undertaken tests on more occasions than others (between one and seven occasions), we also wanted to control for the potentially disproportionate effect of inter-individual differences. We therefore used a linear mixed-effect regression model with random effects attributed to individual patients and fixed effects to the four covariates listed above. We used the $\mathrm{R}$ statistical package with the library lme4 (function lmer). As a secondary analysis we compared the first and last cognitive tests of those patients who had 13 or more ECT sessions between these tests (more than a standard ECT course of 12 sessions). We used a paired samples $t$-test to compare these pre- and post-ECT results.

\section{Results}

Results are presented in Table 1. We show the effect size and statistical significance of the effect from the number of ECT sessions and the four covariates on cognitive performance. Effect size denotes the change on the cognitive score with each unit of the covariate (such as each year of age or each ECT session). Deterioration is always indicated with a negative sign of the effect size, whether it refers to reduction in score (for example verbal fluency), or increase in the time needed to perform a test (Trail Making and Reaction Time), or increase in the Cognitive Failures Questionnaire (CFQ) score. ${ }^{9,11}$ The number of ECT sessions prior to testing had no significant effect on any of the tests. The other covariates had statistically significant effects on the results, in the predicted directions.

The variable with the strongest effect on the tests was age, with performance significantly declining with increasing age on all tests except the CFQ. The CFQ is a self-assessment of cognitive/ memory problems and the results suggested that older patients complained less of cognitive problems. The next factor influencing the results was the severity of depression at the time of testing, which adversely (and significantly) affected seven of the nine tests.

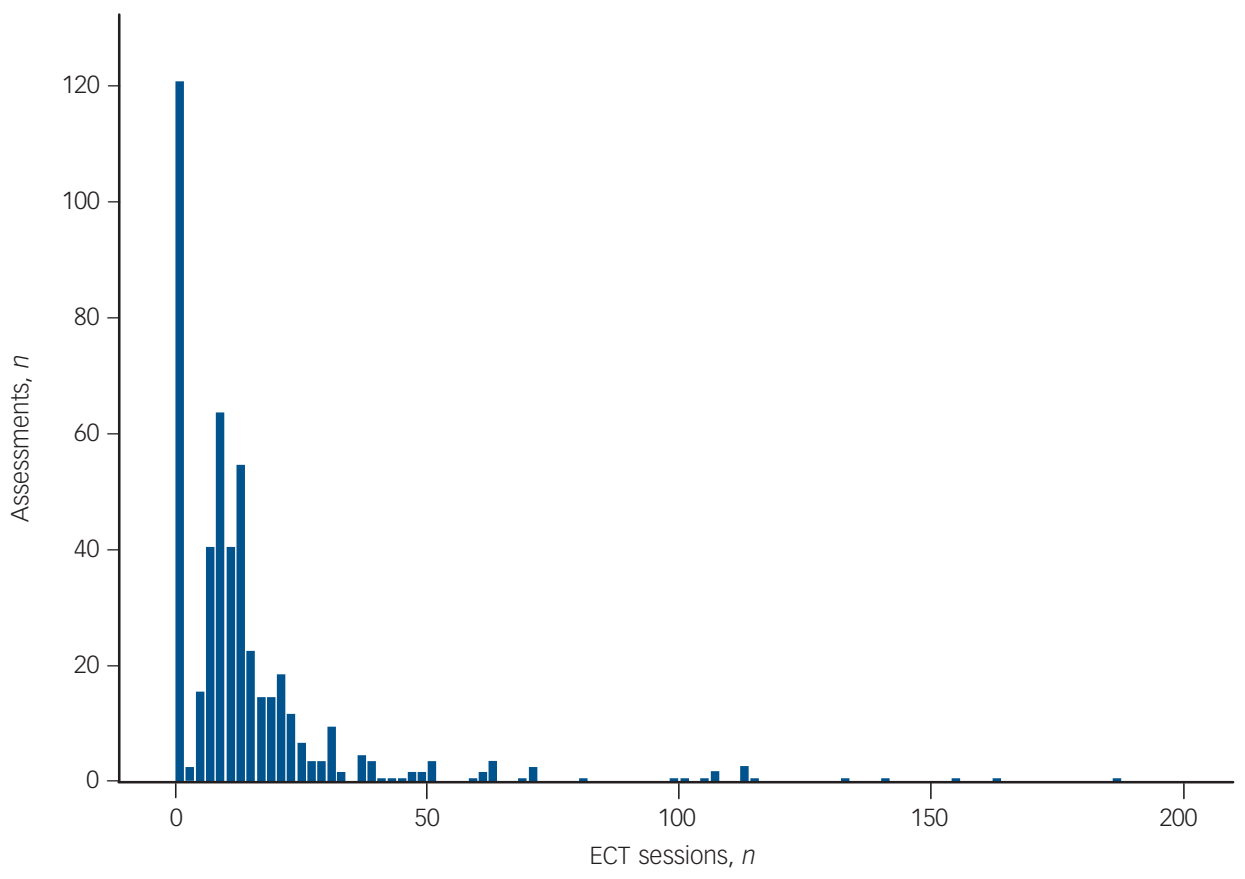

Fig. 1 Number of electroconvulsive therapy (ECT) sessions received by patients prior to the assessments. 


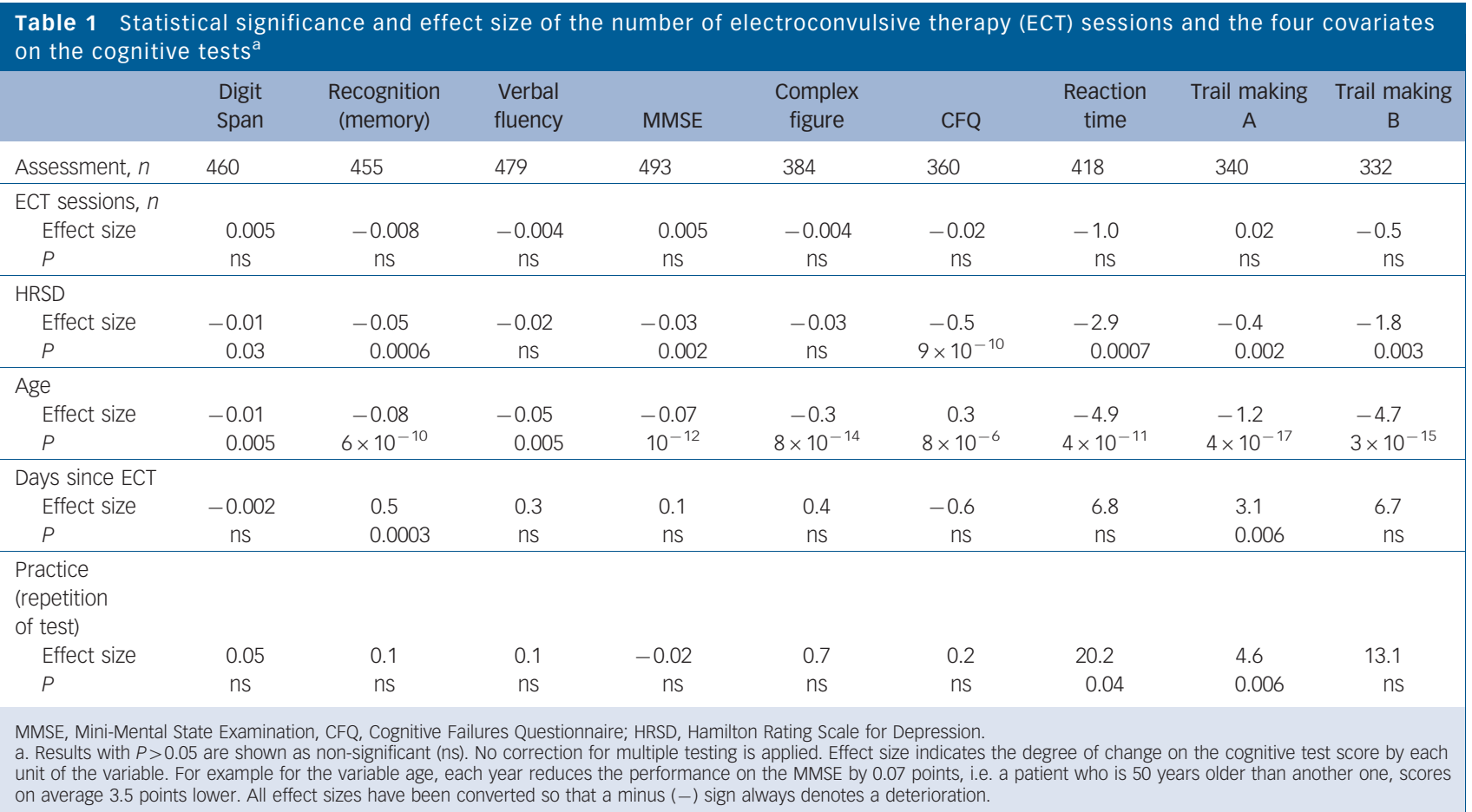

Patients showed significant improvements in performance with increasing time-gaps since the last ECT session on two of the tests (memory and Trail Making A). Practice (repetition of the tests) significantly improved the performance on two tests (Reaction Time and Trail Making A).

As a secondary analysis we compared the pre- and post-ECT results on 55 patients who had 13 or more ECT sessions between their first and last cognitive assessment (i.e. more than the standard duration of a single ECT course of up to 12 sessions). The median number of ECT sessions these patients had between the tests was 20 (range 13-156). We used paired samples $t$-tests and present the results in Table 2. All results improved, two of them improved significantly. The improvements are most likely because of improvements in depression at the end of a course or at follow-up (HRSD-24 scores were on average 29.1 at the first and 14.7 at the time of the last tests).

\section{Discussion}

\section{Findings from other studies}

Memory problems and fears of cognitive decline are probably the biggest deterrents for patients' willingness to undergo ECT. ${ }^{15}$ The internet abounds with reports of people complaining that ECT has destroyed their memory and mainstream media tends to portray it as a barbaric treatment. ${ }^{7}$ Complaints of memory loss regarding events occurring around, or a few months prior to ECT courses (retrograde amnesia) are very frequent and were also reported by many of our patients (the tests in the Appendix do not measure retrograde amnesia). However, a meta-analysis ${ }^{9}$ showed that the reduced performance on various cognitive tests is limited to only the first few days after a ECT session, and tends to improve when such tests are performed more than 2 weeks after the last session. However, the improvement of depression after ECT could have improved performance and masked a small underlying cognitive deterioration (we were able to control for depression severity in the current study). There is less evidence as to whether repeated courses of ECT, or maintenance ECT given over several years, will result in cumulative cognitive deficits. It is conceivable that some small damage is caused by each ECT, which could be overlooked if testing is limited to 3-12 sessions, the typical duration of the majority of ECT courses included in the meta- analysis. ${ }^{9}$ It is impractical to conduct such studies as part of masked, control trials, as they require years of observations and testing patients who might lose capacity to provide informed consent at some periods of their treatments. Most of the evidence on long ECT courses is from case reports and small case series and they show lack of cumulative deterioration. Case reports by Wijkstra \& Nolen ${ }^{16}$ on a patient who received 244 ECT sessions over 7 years, Barnes et a ${ }^{17}$ on a patient who had 420 ECT sessions and Zisselman et $a^{18}$ on a patient who had 12 months of weekly maintenance ECT found no evidence of progressive deterioration on the Mini-Mental Sate Examination (MMSE). Russell et al ${ }^{19}$ conducted a retrospective chart review of MMSE scores of 43 patients who had received at least 1 year of maintenance ECT and found a small, not significant improvement in the mean scores. Abraham et al ${ }^{20}$ tested memory changes in 18 patients who had received at least 3 months of maintenance ECT. 'Mild memory impairment' affected just three patients, whereas severe memory changes were only found in one patient. Vothknecht et $a l^{21}$ reported on 11 patients who underwent maintenance ECT and who received sessions on average 2.2 weeks apart and who were tested with a battery of neuropsychological tests at baseline and after 6 months. The results actually improved after 6 months of maintenance ECT. It has been suggested that the risk of cognitive impairment during longer courses of maintenance ECT may even decrease, because of the longer time intervals between sessions. $^{22}$

\section{Main findings}

To our knowledge, the current study is the first of its kind, reporting on 10 years of cognitive data collection on consecutive ECT patients, where each patient's lifetime number of ECT sessions has been recorded and included in the analysis. Our results show a lack of cumulative cognitive deterioration with an increasing number of ECT sessions. In contrast, other covariates, such as age and severity of depression, produced clear trends in the expected directions, suggesting that we have not missed trends 


\begin{tabular}{|c|c|c|c|c|c|}
\hline Test & Patients, $n$ & Start (s.d.) & End (s.d.) & Change $(95 \% \mathrm{Cl})$ & $P$ \\
\hline Digit span backwards & 50 & $4.4(1.3)$ & $4.7(1.3)$ & $0.3(-0.02$ to 0.7$)$ & 0.06 \\
\hline Recognition of words, shapes and faces (memory, maximum 28) & 48 & $23.4(3.7)$ & $23.7(3.2)$ & $0.3(-0.5$ to 1.1$)$ & 0.5 \\
\hline Verbal fluency & 52 & $10.7(4.3)$ & $11.0(4.6)$ & $0.3(-1.1$ to 1.6$)$ & 0.7 \\
\hline Mini-Mental State Examination (maximum 30) & 55 & $27.7(3.0)$ & $27.7(2.9)$ & $0.0(-0.6$ to 0.7$)$ & 1 \\
\hline Complex Figure immediate recall (maximum 36) & 40 & $20.7(11.3)$ & $21.7(8.2)$ & $1.0(-1.9$ to 4.0$)$ & 0.5 \\
\hline Cognitive Failures Questionnaire (CFQ) & 31 & $50.1(19.0)$ & $42.1(17.0)$ & $-8.0(-13.4$ to -2.5$)$ & 0.006 \\
\hline Reaction time, $\mathrm{s}$ (maximum $1.1 \mathrm{~s}$ ) & 43 & $0.66(0.23)$ & $0.54(0.16)$ & $-0.12(-0.18$ to -0.05$)$ & 0.001 \\
\hline Trail making A, s (maximum $300 \mathrm{~s}$ ) & 32 & $56.8(47.9)$ & $51.0(50.7)$ & $-5.8(-13.0$ to 1.4$)$ & 0.1 \\
\hline Trail making B, s (maximum $600 \mathrm{~s}$ ) & 32 & $119.4(117.4)$ & $111.2(121.0)$ & $-8.2(-30.8$ to 14.3$)$ & 0.5 \\
\hline
\end{tabular}

where they exist, because of noise in the data or bias that can occur in an open study. An example of this is presented in Fig. 2 that shows a clear deterioration of the scores on the Complex Figure test ${ }^{12}$ with increasing age of the patients, but there is no such trend with increasing number of ECT sessions. The results are supported by our observations on patients who accumulated the highest numbers of ECT sessions over the 10-year period of monitoring and showed no deterioration in their performance (Table 2).

(a)

(a) $38-$
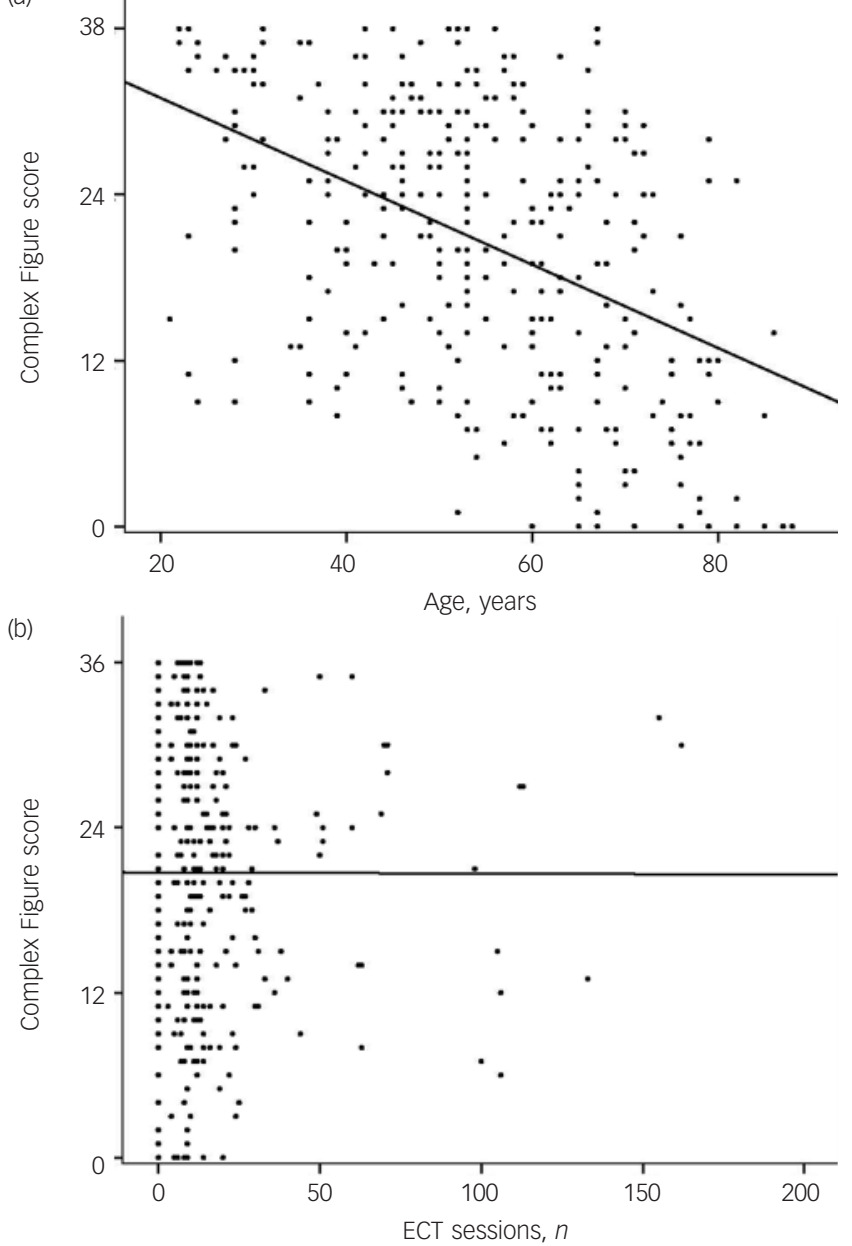

Fig. 2 Scores on the Complex Figure immediate recall test.

The score on the complex Figure immediate recall test reduces with the increasing age of patients (a) but not with the increasing number of previous electroconvulsive therapy (ECT) sessions (b). The maximum score for a perfectly memorised and reproduced figure is 36 points. ${ }^{12}$

\section{Limitations}

As an open, uncontrolled study, our work has limitations that we acknowledge. For example, some patients might have had early stages of dementia or underlying cerebrovascular disease, which could lead to a reduced cognitive reserve. To reduce this potential problem, we re-analysed the results while excluding people over the age of 65. The lack of effect from the number of ECT sessions remained for all cognitive tests (data not presented). The effect size caused by age was reduced, as expected, but remained in the same direction. It is also possible that the patients who persist with more ECT sessions may be those who had fewer cognitive side-effects, so that those who have more ECT sessions will tend to have fewer adverse effects. This potential bias is difficult to address in a naturalistic study, and we can only report that many of our patients on maintenance ECT or repeated courses complained of subjective memory problems and stopped their treatments on many occasions, i.e. they did not appear to be more tolerant of side-effects. We were unable to measure any cumulative retrograde amnesia because of the longitudinal nature of the testing (patients are more likely to forget the original events, if tested 1 or more years later, when they come for new ECT courses).

\section{Implications}

We find that discussing the cognitive results with patients is highly reassuring for them, as they can see for themselves that their cognitive performance remains stable. This has helped their decisions when accepting further ECT courses or maintenance treatment, something that might have been unacceptable to them or to their treatment teams only a few years ago. Our results suggest that patients can have repeated courses or maintenance ECT without this resulting in long-term cumulative cognitive deterioration. We hope that this study will help to counter the negative public view of ECT, which may be having an effect on prescribers and thus aid in the appropriate prescribing of this effective treatment.

George G. Kirov, MRCPsych, PhD, MRC Centre for Neuropsychiatric Genetics \& Genomics, Cardiff University, Cardiff; Laura Owen, MBBCh, Intensive Care Unit, Department of Anaesthetics, Royal Gwent Hospital, Newport; Hazel Ballard, MRC Centre for Neuropsychiatric Genetics \& Genomics, Cardiff University, Cardiff; Adele Leighton, MBBCh, Kara Hannigan, RGN, Danielle Llewellyn, BN, Cardiff \& Vale University Health Board, Whitchurch Hospital, Cardiff; Valentina Escott-Price, PhD, MRC Centre for Neuropsychiatric Genetics \& Genomics, Cardiff University, Cardiff; Maria Atkins, MRCPsych, Cardiff \& Vale University Health Board, Whitchurch Hospital, Cardiff, UK

Correspondence: George Kirov, Cardiff University, Hadyn Ellis Building, Heath Park, Cardiff CF24 4HQ, UK. Email: kirov@cardiff.ac.uk

First received 22 Oct 2014, final revision 7 Mar 2015, accepted 13 Mar 2015 


\section{Appendix}

\section{List of cognitive tests and the functions they measure ${ }^{a}$}

\begin{tabular}{|c|c|c|}
\hline Test & Description of the test & Cognitive domain tested \\
\hline Digit Span backwards & Number of digits repeated backwards (four versions) & Concentration, attention and working memory \\
\hline Recognition of words, shapes and faces & $\begin{array}{l}\text { Recognising } 12 \text { words, } 8 \text { shapes and } 8 \text { faces presented } \\
20-30 \text { min earlier }\end{array}$ & Verbal and visual episodic (anterograde) memory \\
\hline Verbal fluency & $\begin{array}{l}\text { Naming words beginning with a given letter in } 1 \mathrm{~min} \\
\text { (four versions: } F, S, T, A \text { ) }\end{array}$ & Executive functioning and linguistic ability \\
\hline Mini Mental State Examination (MMSE) & Four versions adapted from the standard MMSE & Global cognitive functioning and orientation \\
\hline Complex Figure immediate recall & Complex figure to be drawn from memory (four versions) & Visual episodic memory and executive functioning \\
\hline Cognitive Failures Questionnaire (CFQ) & $\begin{array}{l}\text { Self-rating on a series of } 25 \text { statements about everyday } \\
\text { cognitive/memory problems }\end{array}$ & $\begin{array}{l}\text { Subjective assessment of memory and cognitive } \\
\text { performance }\end{array}$ \\
\hline Reaction time & $\begin{array}{l}\text { Speed of reactions assessed with a computer test } \\
\text { (maximum time limited to } 1.1 \mathrm{~s} \text { ) }\end{array}$ & Processing speed \\
\hline Trail making A & $\begin{array}{l}\text { The time needed to connect } 24 \text { scattered numbers in } \\
\text { ascending order (maximum time limited to } 5 \text { min) }\end{array}$ & Processing speed, visual scanning, mental flexibility \\
\hline Trail making B & $\begin{array}{l}\text { The time needed to connect } 12 \text { scattered numbers and } \\
12 \text { letters in order (maximum time limited to } 10 \mathrm{~min} \text { ) }\end{array}$ & $\begin{array}{l}\text { Executive functioning, visual scanning, mental } \\
\text { flexibility }\end{array}$ \\
\hline
\end{tabular}

\section{References}

1 UK ECT Review Group. Efficacy and safety of electroconvulsive therapy in depressive disorders: a systematic review and meta-analysis. Lancet 2003; 361: 799-808.

2 Dierckx B, Heijnen WT, van den Broek WW, Birkenhäger TK. Efficacy of electroconvulsive therapy in bipolar versus unipolar major depression: a meta-analysis. Bipolar Disord 2012; 14: 146-50.

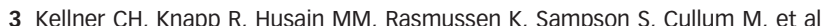
Bifrontal, bitemporal and right unilateral electrode placement in ECT: randomised trial. Br J Psychiatry 2010; 196: 226-34.

4 Petrides G, Fink M, Husain MM, Knapp RG, Rush AJ, Mueller M, et al. ECT remission rates in psychotic versus nonpsychotic depressed patients: a report from CORE. J ECT 2001; 17: 244-53.

5 Heijnen WT, Birkenhäger TK, Wierdsma Al, van den Broek WW. Antidepressant pharmacotherapy failure and response to subsequent electroconvulsive therapy: a meta-analysis. J Clin Psychopharmacol 2010; 30: 616-9

6 Prudic J, Olfson M, Marcus SC, Fuller RB, Sackeim HA. Effectiveness of electroconvulsive therapy in community settings. Biol Psychiatry 2004; 55: 301-12.

7 Byrne P. Now this won't hurt a bit - psychiatry in the movies. Br J Psychiatry 2010; 196: 411.

8 Semkovska M, McLoughlin DM. Measuring retrograde autobiographical amnesia following electroconvulsive therapy: historical perspective and current issues. J ECT 2013; 29: 127-33.

9 Semkovska M, McLoughlin DM. Objective cognitive performance associated with electroconvulsive therapy for depression: a systematic review and meta-analysis. Biol Psychiatry 2010; 68: 568-77.

10 Kirov G, Ebmeier KP, Scott Al, Atkins M, Khalid N, Carrick L, et al. Quick recovery of orientation after magnetic seizure therapy for major depressive disorder. Br J Psychiatry 2008; 193: 152-5.
11 Lezak MD, Howieson DB, Loring DW. Neuropsychological Assessment (4th edn). Oxford University Press, 2004

12 Loring DW, Meador KJ. The Medical College of Georgia (MCG) Complex Figures: four forms for follow-up. In The Handbook of Rey-Osterrieth Complex Figure Usage: Clinical and Research Applications (eds JA Knight, E Kaplan): 313-21. Psychological Assessment Resources, 2003.

13 Heinrichs RW, Zakzanis KK. Neurocognitive deficit in schizophrenia: a quantitative review of the evidence. Neuropsychol 1998; 12: 426-45.

14 Blacker D. Psychiatric rating scales. In Comprehensive Textbook of Psychiatry (9th edn) (eds BJ Sadock, VA Sadock, P Ruiz): 1032-59. Lippincott Williams and Wilkins, 2009.

15 American Psychiatric Association. A Task Force Report of the American Psychiatric Association. The Practice of Electroconvulsive Therapy: Recommendations for Treatment, Training, and Privileging (2nd edn). APA, 2001.

16 Wijkstra J, Nolen AN. Successful maintenance electroconvulsive therapy for more than seven years. J ECT 2005; 21: 171-3.

17 Barnes RC, Hussein A, Anderson DN. Maintenance electroconvulsive therapy and cognitive function. J Psychiatry 1997; 170: 285-7.

18 Zisselman $\mathrm{MH}$, Rosenquist $\mathrm{PB}$, Curlik SM. Long-term weekly continuation electroconvulsive therapy: a case series. J ECT 2007; 23: 274-7.

19 Russell JC, Rasmussen KG, O'Connor MK, Copeman CA, Ryan DA, Rummans TA. Long-term maintenance ECT: a retrospective review of efficacy and cognitive outcome. J ECT 2003; 19: 4-9.

20 Abraham G, Milev R, Delva N, Zaheer J. Clinical outcome and memory function with maintenance electroconvulsive therapy: a retrospective study. J ECT 2006; 22: 43-5.

21 Vothknecht $\mathrm{S}$, Kho $\mathrm{KH}$, van Schaick HW, Zwinderman AH, Middelkoop $\mathrm{H}$, Blansjaar BA. Effects of maintenance electroconvulsive therapy on cognitive functions. J ECT 2003; 19: 151-7.

22 Trevino K, McClintock M, Husain M. A review of continuation electroconvulsive therapy. J ECT 2010; 26: 186-95. 